\title{
Improving prompt access to malaria diagnostics and treatment in rural remote areas using financial benefit for community health workers in Kilosa district, Tanzania
}

This article was published in the following Dove Press journal:

Research and Reports in Tropical Medicine

\section{Daudi Omari Simba' \\ Deodatus Kakoko \\ Tumaini Nyamhanga ${ }^{3}$ \\ Zakayo Mrango ${ }^{4}$ \\ Phare Mujinja ${ }^{2}$}

'Department of Community Health, School of Public Health and Social Sciences, Muhimbili University of Health and Allied Sciences, Dar-es Salaam, Tanzania; ${ }^{2}$ Department of Behavioural Sciences, School of Public Health and Social Sciences, Muhimbili University of Health and Allied Sciences, Dar-es Salaam, Tanzania; ${ }^{3}$ Department of Development Studies, School of Public Health and Social Sciences, Muhimbili University of Health and Allied Sciences, Dar-es Salaam, Tanzania; ${ }^{4}$ National Institute for Medical Research, Kilosa Station, Kilosa, Tanzania
Correspondence: Daudi Omari Simba Department of Community Health, School of Public Health and Social Sciences, Muhimbili University of Health and Allied Sciences, P.O. Box 650I5,

Dar-es Salaam, Tanzania

Tel +25575 4382209

Fax +255 222153114

Email daudisimba@yahoo.com
Purpose: Improving access to malaria treatment in rural remote areas remains a major challenge facing innovative strategies, such as Accredited Drug Dispensing Outlets (ADDOs) and Community Health Workers (CHWs) programs in Tanzania. This study tested the effectiveness of a financial benefit approach to motivate $\mathrm{CHWs}$ to improve prompt access to malaria treatment. Patients and methods: We applied a quasi-experimental study design in rural-remote areas in Kilosa district, Tanzania. Febrile children in selected intervention areas were provided access to malaria diagnostic and treatment at a minimal fee to $\mathrm{CHWs}$ and compared with non-intervention areas. We measured impact using difference in differences (DID) analysis.

Results: At baseline, 870 children $<5$ years of age were recruited and 1,127 in post-intervention. The DID in prompt access to malaria diagnostics and treatment was $28.0 \%$ in favor of intervention. A net pre and post decrease $(\mathrm{DID}=24.1 \%)$ in seeking care from public facilities was observed, signifying decrease in workload. Incidentally, knowledge on malaria treatment increased in intervention area (DID 11\%-21\%).

Conclusion: Using the financial benefit approach, CHWs were able to significantly improve prompt access to malaria diagnostics and treatment in rural remote areas. Scaling up of the strategy might speed up the pace toward achieving national target of accurate diagnosis and appropriate treatment by $80 \%$ in 2020 .

Keywords: financing, artemether-lumefantrine, malaria, children under-five years, rural areas, prompt access, community health workers

\section{Introduction}

Sub-Saharan African countries contribute $>95 \%$ of the global malaria morbidity and mortality. ${ }^{1}$ Despite a wide coverage of malaria interventions, the decline in disease burden is lowest in these countries. ${ }^{1}$ Reducing mortality through prompt diagnosis and treatment is the cornerstone for the control of malaria ${ }^{2}$ by taking advantage of simpleto-perform malaria rapid diagnostic test (mRDT) and highly efficacious artemisinin combination therapies (ACTs).

The WHO recommends prompt treatment, within 24 hours of the onset of fever, with recommended anti-malarial medicines, after confirmation of malaria using appropriate diagnostic tests. ${ }^{3}$ Prompt access is fundamental to the reduction of malaria mortality among children $<5$ years of age. ${ }^{33}$

Delayed fever treatment is a major bottleneck in achieving the global target of reducing malaria mortality by half. ${ }^{4}$ Studies done in Tanzania reveal that less than 
half of febrile children are reported to receive ACT within 24 hours across Sub-Saharan Africa. ${ }^{5,6}$ Other factors limiting prompt access include distance to public health facilities (PHFs), availability of ACTs, cost of transport, affordability of diagnostics and ACTs, inadequate number and unskilled staff, inadequate quality of health care services, and poor prescribing habits. $^{7-9}$

Global efforts to increase access to ACT, such as subsidization of artemether-lumefantrine ( $\mathrm{ALu}$ ) have made considerable impact. ${ }^{10}$ However, the pace toward attaining universal access to ACT has been quite slow, ${ }^{11}$ particularly with regard to people in rural areas where poverty level and malaria burden are higher ${ }^{12}$ but access to means of malaria diagnosis and treatment are lower. ${ }^{13}$ This renders national strategies to improve access unlikely to be achieved. ${ }^{14}$

In Tanzania, access to ACT is still low despite the decline in malaria morbidity and mortality. This implies that more could be achieved if the drugs' potential was optimized through effective coverage. ${ }^{5,14}$ Since the drug efficacy is high, the major challenge that remains is finding effective ways to deliver these drugs to those who need them most. ${ }^{15}$ Effort to reach the needy, majority of whom reside in rural areas includes deployment of Community Health Workers (CHWs) to deliver some essential and life-saving services. ${ }^{16}$ However, these efforts are challenged by a high CHWs' dropout rate apparently for lack of incentives. ${ }^{17}$ Efforts to improve access to essential and lifesaving medicine in rural areas included the involvement of the private sector through the introduction of Accredited Drug Dispensing Outlets (ADDOs) program. ${ }^{5,18}$ Despite the success in country-wide scaling up, ${ }^{10}$ the ADDOs program has not been able to adequately contribute to ensuring prompt access to anti-malarials in rural and hard to reach areas. ${ }^{5}$ This is partly due to majority of them being located in semi-urban areas. ${ }^{19}$

Innovative intervention is therefore needed to address the problem of high dropout rate among CHWs and extend ADDOs services to rural remote areas in order to improve prompt access to diagnostic and effective malaria treatment among children $<5$ years of age in rural remote areas. We designed a strategy to specifically address these challenges.

\section{Study objective}

This intervention strategy aimed to improve prompt access to diagnostics and treatment of uncomplicated malaria among children $<5$ years of age in rural remote areas.

\section{Hypothesis}

Improving availability of malaria diagnostic and treatment services in rural remote areas using this strategy would result in prompt access to malaria diagnostics and treatment among children $<5$ years of age suffering from uncomplicated malaria.

\section{Materials and methods Study design}

This was a quasi-experimental study performed in 20132014, with before and after intervention cross-sectional studies in an intervention and a non-intervention area.

\section{The intervention strategy}

The strategy involved orientation of stakeholders that included health facility workers in August 2013, ADDO providers, and village leaders, on the objectives and implementation plan of the intervention. This was followed immediately by a baseline study and CHWs training. Prior to intervention, an advocacy campaign was conducted with support from village and hamlet chairpersons to motivate caretakers to take children to the CHWs whenever they get fever. CHWs were deployed to provide services, including testing for malaria parasites using mRDT for children brought with symptoms of fever. Intervention lasted for 12 months, climaxing with a post-intervention study. We took advantage of

1. Existence of CHWs living within the villages: thus, they are available all the time, including on weekends and during nights when most PHFs are closed. ${ }^{20} \mathrm{CHWs}$ also foster equity through preferentially reaching the poorest with their services..$^{20,21}$

2. Country-wide scaling up of the ADDO program to enlist the services of ADDO providers to sell mRDT and ALu to $\mathrm{CHWs}$ at market price. In turn, CHWs sold the same to caretakers at a small profit margin.

3. Organized and functioning village leadership to take their febrile children to the $\mathrm{CHW}$ s and the rationale for paying for the services.

\section{Study setting}

The study was conducted in Kilosa district between August 2013 and September 2014. About one-third of its population lives below 1 dollar per day. The level of poverty was higher in rural areas. Kilosa had a population of 473,180 , of whom 230,346 were males and 242,834 were females, living in 118 villages. ${ }^{22}$ There were 67 health facilities -56 dispensaries, 8 health centers, and 3 hospitals. It had 244 drug shops, which were converted to ADDOs. Among health facilities, faith-based organizations owned 9 dispensaries, 1 health center, and 2 hospitals. Malaria is one of the leading 
causes of outpatient attendance and admissions in the district, accounting for more than half $(55.5 \%)$ of outpatient attendance, and $60 \%$ of deaths among children $<5$ years of age admitted to the hospitals ${ }^{24}$ (District Annual Report, 2014). Rural villages were purposely selected because of the high malaria burden $-10 \%$ in rural vs $3 \%$ in urban. ${ }^{23}$

Intervention and non-intervention areas: Masanze division had a population of $41,000^{22}$ residing in 17 villages comprising 102 hamlets (as explained in Definitions). The division was served by 7 government-owned dispensaries and Kimamba division had 51,000 people residing in 17 villages comprising 83 hamlets. There were 9 dispensaries ( 5 government and 4 private) and 1 health center (government). The main occupation in both divisions was agriculture and livestock keeping.

\section{Sample size determination and sampling} Implementation of the strategy was done in Masanze, 1 of the 7 divisions in Kilosa district. Kimamba division was selected as non-intervention area. The 2 divisions were purposely selected because of their proximity to Kilosa town where the study site was located. All hamlets situated $\geq 5 \mathrm{~km}$ from the nearest PHFs or ADDO were involved. Thirty-three hamlets qualified in the intervention area and 30 in the nonintervention one.

The sampling unit was children $<5$ years of age. Using the formula $n=z 2 p(1-p) / e 2$ where $n=$ sample size, $p=$ proportion of children $<5$ years of age, and e=margin of error/CI, we assumed that $50 \%$ had access to ALu and $95 \% \mathrm{CI}$, the unadjusted sample size for independent observations was found to be 384 children. Adding 10\% for possible loss to follow-up and another $38 \%$ as correction factor for clustering effect (ICC $=0.01$ ), the total sample size was 583 , approximated to 600 in the intervention and another 600 in the non-intervention villages. The number of children from each hamlet was determined using hamlet population proportions. Using systematic sampling, a starting point was selected and the direction to follow was determined by spinning a bottle. With the help of the CHWs, households with children $<5$ years were identified. Random selection was done to select one child where $>1$ was found. During data collection, we found fewer number of children $<5$ years of age in both intervention and non-intervention groups than the sample size. We then decided to enroll all the children $<5$ years of age.

Inclusion and exclusion criteria: at baseline, children aged 3-59 months and whose caretakers were residents of the respective hamlets, for the past $\geq 6$ months, were enrolled in the study; children $<2$ months of age were excluded. ALu is currently not recommended for infants $<2$ months of age (approximately $<5 \mathrm{~kg}$ ).

\section{Data collection procedure}

Two research assistants (RAs) were recruited from the National Institute for Medical Research (NIMR) site at Kilosa to collect data. They received a 2-day training on the study objectives and administering research tools. A pilot test was done in 1 hamlet near the training center to perfect the tools and for RAs to practice on the use of the tools. Hamlet chairperson guided the RAs and introduced them to the selected households. The household head or spouse or any other wellinformed household member was interviewed after obtaining an informed written consent. The RAs collected data using a structured questionnaire. Caretakers were interviewed at their homes to obtain demographic and socio-economic data; and knowledge, attitude, perception, and practice related to the access to ACT. Although presumptive treatment is no longer recommended by WHO, this study classified a child as having malaria if the caretaker reported the child had fever within the past 2 weeks. Most children found to have fever were treated for malaria without confirmation with mRDT due to unavailability of diagnostic facilities. ${ }^{24}$ Anecdotal reports during implementation of this study indicate that some children were referred by health workers to CHWs for mRDT due to frequent shortages of PHFs. Despite some challenges on its interpretation, ${ }^{25}$ prompt access to malaria diagnostics and treatment was assessed by whether treatment was started on the same day or a day after the onset of fever.

Caretakers whose children had history of fever were interviewed about the source of care sought and treatment received. Verification of drug used was done using exercise books used to record medical notes. Where these books were not available, drug containers/packets were examined to identify the type of drug provided. We established knowledge of malaria by asking questions on the cause of malaria, method of transmission, prevention, and treatment.

\section{Data analysis}

Questionnaires were filed and stored at the Kilosa NIMR site. Coding was done and double entered into EpiData 3.0. Analyses were performed using STATA version 9.0.

Dependent variables and measurements:

- Proportion of children whose caretakers sought care from PHFs, including public, private, and health facilities owned by governmental organizations (NGOs), drug stores (ADDO); drug vendors, and ordinary shops 
- Proportion of children whose caretakers accessed appropriate treatment $(\mathrm{ALu})$

- Proportion of children whose caretakers accessed the effective treatment promptly.

Independent variables:

- Socio-demographic data (age, sex, marital status, education, and the main occupation of the mother/guardian), age of the child;

- Source of treatment: includes public, private, and health facilities owned by NGOs, drug stores (ADDO), drug vendors, and ordinary shops;

- Type of drug used: included ACT, other anti-malarials, and antipyretics;

- Socio-economic status was measured using household assets and categorized into quintiles using principal component analysis. ${ }^{6}$ Membership to health insurance schemes: available schemes are Community Health Fund (CHF) and National Health Insurance Fund;

- Knowledge of malaria was measured by asking questions on the cause of malaria, method of transmission, prevention, and treatment. ${ }^{6}$

\section{Analyses}

We calculated the proportion of children whose caretakers: 1) sought care from PHFs; 2) accessed appropriate treatment (mRDT and ALu); and 3) accessed the treatment promptly in the intervention and non-intervention areas.

We applied the difference in differences (DID) analysis to calculate the difference attributed by the intervention using the following formula ${ }^{26}$ :

$$
\left.\left(\mathrm{I}_{\mathrm{P}}-\mathrm{I}_{\mathrm{B}}\right)-\mathrm{N}_{\mathrm{P}}-\mathrm{N}_{\mathrm{B}}\right)
$$

Where I = Intervention area, $\mathrm{N}=$ Non-intervention area, ${ }_{\mathrm{P}}=$ post intervention, and ${ }_{\mathrm{B}}=$ Baseline.

Using the same formula, we calculated the difference in knowledge between intervention and non-intervention areas. Strength of association was measured using chi-square tests and Fisher's exact test (for tables where cell values were $<5$ ). $P$-values $<0.05$ were considered significant. Multivariate analyses were done to control for confounding factors. The AOR and $95 \% \mathrm{CI}$ are used to report the magnitude of association between dependent and independent variables.

\section{Ethical considerations}

Non-clinical workers have previously been used in homebased care treatment of febrile illnesses, including the use of
RDT for malaria..$^{27,28}$ This is because, in the absence of such services, children residing in rural remote areas might not get prompt access to malaria diagnostics and treatment, thus predisposing them to avoidable deaths and malaria complications. ${ }^{29,30}$ The research proposal received ethical clearance from the Muhimbili University of Health and Allied Sciences, Tanzania. Permission to implement the research was obtained from the regional, district, and village authorities. Informed consent was sought from the caretakers of the children.

\section{Results}

\section{Socio-economic and demographic comparison}

A total of 870 children were enrolled in the baseline study, of whom 467 (53.7\%) were from the intervention and 403 (46.3\%) from non-intervention areas. During post-intervention, we enrolled 1,127 children $<5$ years of age of whom 611 (54.2\%) were from the intervention and 516 (45.8\%) from the non-intervention areas. The mean age of the children was 30 months. Over half of the enrolled children were $\geq 2$ years of age and about half of them were females. The mean age of the caretakers was 29.6 years and over half of them were $<30$ years of age and married (Table 1). Less than $5 \%$ of the caretakers reported enrollment in the CHF insurance scheme.

There was no statistically significant difference between the intervention and non-intervention areas with regard to child age and sex and caretakers' marital status. However, mothers' education was higher in the non-intervention than in intervention areas $(P<0.001)$. While over half of the mothers in the non-intervention area had primary education level, in the intervention one, less than half had.

\section{Knowledge on malaria treatment}

Over $80 \%$ of the respondents knew that ALu is the recommended treatment for uncomplicated malaria (Table 2). Almost all respondents reported to have heard about a drug for treating malaria. Prior to intervention, knowledge that $\mathrm{ALu}$ is the recommended drug for treating uncomplicated malaria was higher among the respondents in the nonintervention $(90.8 \%)$ than in intervention areas $(85.7 \%)$. This pattern reversed in the post-intervention period with $94.8 \%$ of respondents in the intervention compared with non-intervention areas (88.8\%). The DID was $11.1 \%$ ( $P$-value $<0.05)$. Less than one-third of the respondents agreed that sulfadoxine-pyrimethamine is the recommended treatment for uncomplicated malaria. However, the proportion of caretakers with this misconception was higher among the respondents in the intervention than in non-intervention areas. Again, 
Table I Demographic and socio-economic characteristics

\begin{tabular}{|c|c|c|c|c|c|c|c|c|c|c|}
\hline \multirow{3}{*}{$\begin{array}{l}\text { Variables } \\
\text { Child's age }\end{array}$} & \multicolumn{5}{|c|}{ Pre-intervention } & \multicolumn{5}{|c|}{ Post-intervention } \\
\hline & \multicolumn{2}{|c|}{$\begin{array}{l}\text { Non-intervention } \\
\text { area }\end{array}$} & \multicolumn{2}{|c|}{$\begin{array}{l}\text { Intervention } \\
\text { area }\end{array}$} & \multirow[t]{2}{*}{$P$-value } & \multicolumn{2}{|c|}{$\begin{array}{l}\text { Non-intervention } \\
\text { area }\end{array}$} & \multicolumn{2}{|c|}{$\begin{array}{l}\text { Intervention } \\
\text { area }\end{array}$} & \multirow[t]{2}{*}{$P$-value } \\
\hline & $n-403$ & $\%$ & $n=467$ & $\%$ & & $n=516$ & $\%$ & $\mathrm{n}=670$ & $\%$ & \\
\hline$<2$ years & 169 & 41.9 & 180 & 38.5 & 0.309 & 203 & 39.3 & 289 & 43.1 & 0.189 \\
\hline $2+$ years & 234 & 58.1 & 287 & 61.5 & & 313 & 60.7 & 381 & 56.9 & \\
\hline \multicolumn{11}{|l|}{ Child's sex } \\
\hline Male & 201 & 49.9 & 237 & 50.7 & 0.797 & 259 & 50.2 & 351 & 52.4 & 0.454 \\
\hline Female & 202 & 50.1 & 230 & 49.3 & & 257 & 49.8 & 319 & 47.6 & \\
\hline \multicolumn{11}{|l|}{ Mothers age } \\
\hline$<30$ years & 251 & 62.3 & 266 & 57.0 & 0.111 & 297 & 59.9 & 340 & 50.9 & 0.020 \\
\hline $30+$ years & 152 & 37.7 & 201 & 43.0 & & 219 & 44.2 & 330 & 49.4 & \\
\hline \multicolumn{11}{|l|}{ Mothers' } \\
\hline \multicolumn{11}{|l|}{ education } \\
\hline$<7$ years & 168 & 41.7 & 274 & 58.7 & 0.0001 & 185 & 35.9 & 362 & 54.0 & 0.0001 \\
\hline $7+$ years & 235 & 58.3 & 193 & 41.3 & & 331 & 64.1 & 308 & 46.0 & \\
\hline \multicolumn{11}{|l|}{ Marital status } \\
\hline Not married & 83 & 20.6 & 93 & 19.9 & 0.803 & 198 & 38.4 & 262 & 39.1 & 0.797 \\
\hline Married & 320 & 79.4 & 374 & 80.1 & & 318 & 61.6 & 408 & 60.9 & \\
\hline \multicolumn{11}{|l|}{ Occupation } \\
\hline Farming & 368 & 91.3 & 395 & 84.6 & 0.003 & 451 & 87.4 & 567 & 84.6 & 0.174 \\
\hline Business/livestock & 35 & 8.7 & 72 & 15.4 & & 65 & 12.6 & 103 & 15.4 & \\
\hline \multicolumn{11}{|c|}{ CHF membership } \\
\hline Not member & 388 & 96.3 & 447 & 95.7 & 0.675 & 514 & 99.6 & 635 & 94.8 & 0.0001 \\
\hline Member & 15 & 3.7 & 20 & 4.3 & & 2 & 0.4 & 35 & 5.2 & \\
\hline \multicolumn{11}{|c|}{ Socio-economic status } \\
\hline Poor ( $I$ and 2 ) & 160 & 39.7 & 188 & 40.3 & 0.847 & 231 & 44.8 & 407 & 60.7 & 0.0001 \\
\hline Better off (3-5) & 243 & 60.3 & 279 & 59.7 & & 285 & 55.2 & 263 & 39.3 & \\
\hline
\end{tabular}

Abbreviation: CHF, community health fund.

the pattern reversed post-intervention and the DID between intervention and non-intervention areas was $20.9 \%(P$-value $<0.05$ ). About one-third or less respondents had correct knowledge on the recommended treatment for severe malaria. The DID between intervention and non-intervention area was $11.0 \%$ ( $P$-value $<0.05)$. Incidentally, knowledge on the causes and prevention of convulsion increased dramatically in both areas, however, the difference was not statistically significant for knowledge on the cause of convulsion. After controlling for confounding factors that included mother's age, education and occupation, we found that the intervention had an influence on caretakers' knowledge on recommended treatment for uncomplicated malaria (DID 11.1\% [P-value $<0.05$ ] and AOR 2.01, 95\% CI: 1.26 to 3.15 ).

\section{Care seeking, access and treatment of children $<5$ years of age}

Prior to intervention, a higher proportion of caretakers (59.0\%) in the intervention area sought care from PHFs compared with non-intervention one (44.8\%) (Table 3). However, this pattern reversed during intervention period by having more caretakers from the non-intervention $(57.1 \%)$ than in intervention areas (44.9\%). The DID was $-26.4 \%$ ( $P$-value $<0.05)$, implying that caretakers in the intervention area sought care from PHFs less frequently compared with those from non-intervention one. When controlled for potential confounders, caretakers in the non-intervention area were twice more likely to seek care for their children compared with those in intervention one AOR -1.9 (95\% CI: -1.04 to -3.3$)$.

Over half of the children reported to have had a fever episode were treated with ALu. Whereas, before intervention, the proportion of febrile children treated with ALu was significantly higher in the non-intervention area (71.3\%) compared with intervention one $(53.4 \%)$, this pattern reversed during post-intervention study. The proportion of those treated with $\mathrm{ALu}$ in the intervention area increased from $53.4 \%$ to $70.8 \%$. The DID between the intervention and non-intervention areas was $26.1 \%$. After controlling for potential confounders, children in the non-intervention area were twice more likely to be treated with ALu compared with those in the intervention one AOR 0.5 (95\% CI: 0.3 to 0.8 ) meaning that the observed DID was influenced by the confounding factors. 
Table 2 Knowledge on malaria and treatment

\begin{tabular}{|c|c|c|c|c|c|c|c|c|c|c|c|c|c|}
\hline \multirow[t]{3}{*}{ Variable } & \multicolumn{5}{|c|}{ Pre-intervention } & \multicolumn{8}{|c|}{ Post-intervention } \\
\hline & \multicolumn{2}{|c|}{$\begin{array}{l}\text { Non- } \\
\text { intervention }\end{array}$} & \multicolumn{3}{|c|}{ Intervention } & \multicolumn{2}{|c|}{$\begin{array}{l}\text { Non- } \\
\text { intervention }\end{array}$} & \multicolumn{3}{|c|}{ Intervention } & \multicolumn{3}{|c|}{ DID estimation } \\
\hline & No. & $\%$ & No. & $\%$ & $P$-value & No. & $\%$ & No. & $\%$ & $P$-value & DID\% & $P$-value & AOR $(95 \% \mathrm{Cl})$ \\
\hline \multicolumn{14}{|c|}{ Knowledge that ALu is the recommended treatment for uncomplicated malaria } \\
\hline Spontaneous & 366 & 90.8 & 400 & 85.7 & 0.064 & 458 & 88.8 & 635 & 94.8 & 0.0001 & II.I & 0.0001 & 2.01 \\
\hline response & & & & & & & & & & & & & (1.26 to 3.15$)$ \\
\hline After probe & 31 & 7.7 & 57 & 12.2 & & 46 & 8.9 & 33 & 4.9 & & & & \\
\hline Do not know & 6 & 1.5 & 10 & 2.1 & & 12 & 2.3 & 2 & 0.3 & & & & \\
\hline \multicolumn{14}{|c|}{ Knowledge that SP is the recommended treatment for uncomplicated malaria } \\
\hline Spontaneous & 35 & 8.7 & 85 & 18.2 & 0.0001 & 168 & 32.6 & 142 & 21.2 & 0.0001 & -20.9 & 0.0001 & -2.12 \\
\hline response & & & & & & & & & & & & & $(-1.31$ to $-3.4 \mid)$ \\
\hline After probe & 332 & 82.4 & 330 & 70.7 & & 234 & 45.3 & 354 & 52.8 & & & & \\
\hline Do not know & 36 & 8.9 & 52 & II.I & & 114 & 22.1 & 174 & 26 & & & & \\
\hline \multicolumn{14}{|c|}{ Knowledge that quinine is the recommended treatment for uncomplicated malaria } \\
\hline Spontaneous & 28 & 6.9 & 37 & 7.9 & 0.194 & 85 & 16.5 & 77 & 11.5 & 0.0001 & -6 & 0.0001 & -0.44 \\
\hline response & & & & & & & & & & & & & $(-0.18$ to 0.91$)$ \\
\hline After probe & 304 & 75.4 & 327 & 70 & & 283 & 54.8 & 295 & 44 & & & & \\
\hline Do not know & 71 & 17.6 & 103 & 22.1 & & 148 & 28.7 & 298 & 44.5 & & & & \\
\hline \multicolumn{14}{|c|}{ Which drug is recommended for treating severe malaria? } \\
\hline Others & 323 & 80.1 & 345 & 73.9 & 0.029 & 419 & 81.2 & 429 & 64.0 & 0.0001 & II & 0.0001 & 1.09 \\
\hline & & & & & & & & & & & & & (0.87 to 1.33$)$ \\
\hline Quinine & 80 & 19.9 & 122 & 26.1 & & 97 & 18.8 & 241 & 36.0 & & & & \\
\hline \multicolumn{14}{|c|}{ What causes convulsion in children? } \\
\hline Others & 257 & 63.8 & 281 & 60.2 & 0.276 & 202 & 39.1 & 262 & 39.1 & 0.988 & -3.6 & 0.0001 & -0.20 \\
\hline & & & & & & & & & & & & & $(-0.12$ to 0.32$)$ \\
\hline High fever & 146 & 36.2 & 186 & 39.8 & & 314 & 60.9 & 408 & 60.9 & & & & \\
\hline \multicolumn{14}{|c|}{ How can you prevent a child from getting convulsion? } \\
\hline Others & 398 & 98.8 & 443 & 94.9 & 0.001 & 399 & 77.3 & 550 & 82.1 & 0.042 & -8.7 & 0.0001 & -3.85 \\
\hline & & & & & & & & & & & & & $(-1.16$ to 11.29$)$ \\
\hline $\begin{array}{l}\text { Sponging or } \\
\text { analgesics }\end{array}$ & 5 & 1.2 & 24 & 5.1 & & 117 & 22.7 & 120 & 17.9 & & & & \\
\hline
\end{tabular}

Abbreviations: ALu, artemether-lumefantrine; AOR, adjusted OR; DID, difference in differences; SP, sulfadoxine-pyrimethamine.

Table 3 Care seeking and treatment practices for caretakers with febrile children

\begin{tabular}{|c|c|c|c|c|c|c|c|c|c|c|c|c|c|}
\hline & \multicolumn{5}{|c|}{ Pre-intervention } & \multicolumn{5}{|c|}{ Intervention } & \multirow{2}{*}{\multicolumn{3}{|c|}{ DID estimation }} \\
\hline & \multicolumn{2}{|c|}{$\begin{array}{l}\text { Non- } \\
\text { intervention }\end{array}$} & \multicolumn{3}{|c|}{ Intervention } & \multicolumn{2}{|c|}{$\begin{array}{l}\text { Non- } \\
\text { intervention }\end{array}$} & \multicolumn{3}{|c|}{ Intervention } & & & \\
\hline & No. & $\%$ & No. & $\%$ & $P$-value & No. & $\%$ & No. & $\%$ & $P$-value & DID\% & $P$-value & AOR (95\% Cl) \\
\hline \multicolumn{14}{|c|}{ Seeking care from public health facility by caretakers with febrile children } \\
\hline Total & $\mathrm{n}=105$ & & $\mathrm{n}=117$ & & & $\mathrm{n}=98$ & & $\mathrm{n}=176$ & & & & & \\
\hline No & 58 & 55.2 & 48 & 41.0 & 0.034 & 42 & 42.9 & 97 & 55.1 & 0.052 & $-26.4 \%$ & 0.034 & $-1.9(-1.04$ to 3.3$)$ \\
\hline Yes & 47 & 44.8 & 69 & 59.0 & & 56 & 57.1 & 79 & 44.9 & & & & \\
\hline \multicolumn{14}{|c|}{ Febrile children treated with ALu } \\
\hline Total & $\mathrm{n}=108$ & & $\mathrm{n}=133$ & & & $\mathrm{n}=99$ & & $\mathrm{n}=178$ & & & & & \\
\hline No & 31 & 28.7 & 62 & 46.6 & 0.005 & 37 & 37.4 & 52 & 29.2 & 0.163 & $26.1 \%$ & 0.006 & $0.5(0.3$ to 0.8$)$ \\
\hline Yes & 77 & 71.3 & 71 & 53.4 & & 62 & 62.6 & 126 & 70.8 & & & & \\
\hline \multicolumn{14}{|c|}{ Prompt treatment of uncomplicated malaria (treatment within 24 hours) } \\
\hline Total & $\mathrm{n}=104$ & & $\mathrm{n}=119$ & & & $n=90$ & & $\mathrm{n}=|5|$ & & & & & \\
\hline $24+$ hours & 29 & 27.9 & 41 & 34.5 & 0.292 & 30 & 33.3 & 18 & 11.9 & 0.0001 & $28.0 \%$ & 0.0001 & $2.8(1.4$ to 5.6$)$ \\
\hline$<24$ hours & 75 & 72.1 & 78 & 65.5 & & 60 & 66.7 & 133 & 88.1 & & & & \\
\hline
\end{tabular}

Abbreviations: ALu, artemether-lumefantrine; AOR, adjusted OR; DID, difference in differences. 
At pre-intervention, the proportion of febrile children treated with ALu promptly was significantly higher in the non-intervention area $(72.1 \%)$ compared with intervention one $(65.5 \%)$. This also reversed during post-intervention ( $88.1 \%$ intervention and $66.7 \%$ non-intervention). The DID between intervention and non-intervention areas was $28.0 \%$. Controlled for potential confounders, children in the intervention area were almost 3 times more likely to promptly access ALu compared with those in intervention one (AOR $2.8[1.4$ to 5.6$])$.

\section{Discussion}

This study has shown that using the financial benefit approach for CHWs to treat malaria in children $<5$ years of age can increase prompt access to malaria diagnostics and effective malaria treatment by $28 \%$ ( 3 -fold) in rural remote areas. Over $80 \%$ of the children in the intervention area were able to promptly access effective anti-malarials. Previous studies have shown that $<50 \%$ of children $<5$ years of age accessed appropriate malaria treatment promptly. ${ }^{5,6}$

Achievement reported in this intervention study could be attributed to the financial benefit that might have directly or indirectly motivated CHWs to provide services efficiently and hence, enhanced their gratification, which ensured sustainability. Although CHWs are reportedly engaged to work on voluntary basis, studies show their motivation by material gains. ${ }^{31,32}$ The achievement could also be attributed to the proximity of CHWs to caretakers that ensured timely access to care even during nights and weekends, which is uncommon with PHFs. Moreover, CHWs are more acceptable to the community they serve because they tend to share common values, traditions, and prejudices.

Caretakers in rural remote areas have limited access to ADDO because most ADDOs are situated in semi-urban areas where there is profit gains. ${ }^{19} \mathrm{We}$, therefore, used ADDOs available in intervention areas to supply CHWs with mRDT and $\mathrm{ALu}$, at a market price, thus extending ADDOs reach to a wider geographical area. Procurement through ADDOs ensured maintenance of the supply chain because ADDO providers also work on financial benefit. We deliberately avoided procurement of supplies through the public sector because the system is overwhelmed with frequent shortages. Stockout rates ranging from 69\% to 95\%, were reported between 2011 and 2015 (Ministry of Health-HMIS Database, 2015 - unpublished).

Implementation of this strategy was associated with a 2-fold decrease in the proportion of febrile children taken to PHFs. This could be explained by the presence of CHWs who provided a service that was supposed to be provided by PHFs.
Community-based management of fever in children $<5$ years of age is reported to reduce workload in health facilities ${ }^{33}$ since CHWs shoulder some of the health workers' responsibilities. ${ }^{34}$ Malaria in children $<5$ years of age, accounts for about one-third of outpatient department attendances in malaria hyper-endemic areas in the country ${ }^{35}$ (Ministry of Health - HMIS Database, 2015 - unpublished). This strategy, therefore, has the potential to reduce workload in PHFs.

In this intervention study, we observed a 2-fold increase in knowledge in knowledge on treatment of uncomplicated malaria in the intervention area compared to non-intervention area. This could be due to the multiplier effect resulting from advocacy campaigns held in the villages prior to implementation and the interaction between CHWs and caretakers in the course of service provision.

\section{Implications of intervention}

This intervention increased prompt access to malaria treatment in children residing in rural remote areas. The increase in prompt access is likely to have averted deaths and serious complications commonly associated with delayed treatment. These include increased risk of neurological and cognitive deficits, behavioral difficulties, and epilepsy. ${ }^{30}$ These complications ultimately compromise victims' educational achievements and some of them end up with lifelong dependency and poverty. Scaling up of a similar financial benefit approach is likely to speed up the pace toward attaining the National Malaria Strategic Plan goals. ${ }^{12}$ Implementation research might be needed to explore the feasibility and applicability of this approach in different contexts. Encouragingly, the malaria treatment guidelines and the recent community-based health care policy guidelines provide for $\mathrm{CHWs}$ to treat common conditions, including uncomplicated malaria after testing positive with mRDT (Ministry-of-Health-and-Social-Welfare, 2006).

We do not have data to ascertain the decline in workload in PHFs. However, we can confidently use the significant decrease in utilization of PHFs in the intervention area as enough evidence. Tanzania has been facing a major shortage of health care workers for over a decade, with staffing gap running at nearly half the required numbers. The situation is worse among clinicians in remote and rural areas in PHFs. ${ }^{36}$ Adoption of this strategy might give health facility staff ample time to concentrate on conditions that require more training, hence, increasing productivity and quality of health care. ${ }^{34}$

The selection of Kilosa district and the 2 divisions was purposive. However, findings from this study can be generalized to many other places within and outside the country that are typical rural areas with high malaria endemicity and 
facing considerable health system challenges such as shortage of health workers, medicines and supplies and inadequate number of health facilities.

\section{Methodological considerations}

Ensuring constant supply of mRDT and ALu in PHFs could have reduced the magnitude of observed intervention achievement. However, it is equally true that frequent drug shortages have been there for a considerably long time (Ministry of Health HMIS Database, 2015. Unpublished) and is likely to continue existing in the foreseeable future given the meager budget allocation to the health sector. Since prompt access to effective malaria treatment is an urgent concern, findings from this intervention will remain relevant. Nevertheless, the study could have benefited from a second control in which uninterrupted supply of mRDT and ALu would be ensured but without the financial benefit approach.

Promptness of access was defined by whether or not the child received ALu on the same or next day. This definition suffers from the limitation of extending to over 24 hours..$^{5,25}$ The alternative could be to elicit and record the time of fever onset and ALu treatment from the caretakers. However, this alternative has the limitation of inaccuracy in a community where literacy rate is low. We used fever as a proxy presumption of malaria. However, in areas where malaria has significantly declined, this assumption no longer holds.

The targeted number of 1,200 (600 interventions and 600 non-interventions) could not be attained because, in some of the hamlets, all the children $<5$ years of age were enrolled before reaching the targeted number. Apparently, in some of these hamlets, the number of children found in the census/ village register was higher than the actual. This could be attributed to inadequacies in updating the village register and some children $<5$ years of age might have crossed over the age without being deleted from the registers.

We chose pre-post test quasi-experimental with nonintervention group to control for known and unknown confounders. However, mothers' level of education, during pre-test, was found to be higher in non-intervention than in intervention area. This was associated with increased knowledge on malaria treatment and in prompt access to effective malaria treatment. ${ }^{6}$ Such bias has the effect of understating the effect of the intervention, thus diluting the observed difference. This source of error can be ignored given the significant evidence observed in this study.

We based malaria diagnosis on presumptive treatment in both baseline and post intervention studies. Therefore, we can confidently ascribe the improved access to ALu but not mRDT. However, since all children in the intervention area were treated after diagnosis with mRDT, the improved access to ALu is attributed to the intervention - we can, with caution, generalize our findings to access to mRDT.

\section{Conclusion}

This study has shown that using a financial benefit approach enables CHWs to successfully improve prompt access to diagnostic and effective malaria treatment in areas situated $\geq 5 \mathrm{~km}$ from a nearby PHF or ADDO. Scaling up of the strategy is likely to speed up the pace toward attaining national target of accurate diagnosis and appropriate treatment by $80 \%$ in 2020 .

\section{Definitions}

Prompt access is defined as ability to access appropriate antimalarial treatment within 24 hours from the onset of fever. ${ }^{2}$ In this study, we operationalized the definition based on whether or not the child received ALu on the same or next day.

Community health workers (CHWs) are defined as members of the communities engaged in activities that support the health system. ${ }^{37}$

Rural remote areas are communities located $\geq 5 \mathrm{~km}$ from a nearby health facility or a drug shop.

A hamlet is the smallest administrative unit in the government structure in Tanzania, after the village level. An average hamlet has about 50 households and is led by a hamlet chairperson elected from among members of the respective hamlet. Hamlet chairperson represents the hamlet in the village government meetings.

\section{Acknowledgments}

The authors wish to thank Mr Fransis Mulokozi, Joseph Shishira, and Steve Ngatunga for participating in the study during field work. This study received financial support from Grand Challenge Canada.

\section{Author contributions}

All authors contributed to data analysis, drafting and revising the article, gave final approval of the version to be published, and agree to be accountable for all aspects of the work.

\section{Disclosure}

The authors report no conflicts of interest in this work.

\section{References}

1. WHO. World Malaria Report 2015. 2015. Available from: http://www. who.int/malaria/publications/world-malaria-report-2015/wmr2015without-profiles.pdf?ua=1. Accessed October 3, 2018. 
2. WHO. World Malaria Report 2010. 2010. Available from: http://www. who.int/malaria/world_malaria_report_2010/en/.Accessed October 3, 2018.

3. WHO. World Malaria Report 2014. 2014. Available from: HYPERLINK "http://www.who.int/malaria/publications/world_malaria_report_2014/ wmr-2014-no-profiles.pdf" www.who.int/malaria/publications/world_ malaria_report_2014/wmr-2014-no-profiles.pdf.Accessed October 3, 2018.

4. Alba S, Dillip A, Hetzel MW, et al. Improvements in access to malaria treatment in Tanzania following community, retail sector and health facility interventions -- a user perspective. Malar J. 2010;9:163.

5. Khatib RA, Selemani M, Mrisho GA, et al. Access to artemisinin-based anti-malarial treatment and its related factors in rural Tanzania. Malar J. 2013;12(1):155-158.

6. Simba DO, Warsame M, Kakoko D, et al. Who gets prompt access to artemisinin-based combination therapy? A prospective communitybased study in children from rural Kilosa, Tanzania. PLoS One. 2010;5(8):e12104-12108.

7. Chuma J, Okungu V, Molyneux C. Barriers to prompt and effective malaria treatment among the poorest population in Kenya. Malar J. 2010;9(1):144-14.

8. Iwelunmor J, Idris O, Adelakun A, Airhihenbuwa CO. Child malaria treatment decisions by mothers of children less than five years of age attending an outpatient clinic in south-west Nigeria: an application of the PEN-3 cultural model. Malar J. 2010;9:354.

9. Kizito J, Kayendeke M, Nabirye C, Staedke SG, Chandler CI. Improving access to health care for malaria in Africa: a review of literature on what attracts patients. Malar J. 2012;11:55.

10. Sabot OJ, Mwita A, Cohen JM, et al. Piloting the global subsidy: the impact of subsidized artemisinin-based combination therapies distributed through private drug shops in rural Tanzania. PLoS One. 2009;4(9):e6857.

11. Travis P, Bennett S, Haines A. Overcoming Health-Systems Constraints to Achieve the Millennium Development Goals. Lancet. 364(9437):900-906

12. Ministry-of-Health-and-Social-Welfare. National Malaria Strategic Plan 2014-2020. 2014. Available from: http://ihi.eprints.org/3314/1/ Malaria_Strategic_Plan_Full_Version_02_27_14.pdf. Accessed October 3, 2018.

13. Schellenberg JA, Victora CG, Mushi A, et al. Inequities among the very poor: health care for children in rural southern Tanzania. Lancet. 2003;361(9357):561-566.

14. Siekmans K, Sohani S, Kisia J, et al. Community case management of malaria: a pro-poor intervention in rural Kenya. Int Health. 2013;5(3):196-204.

15. Whitty CJM, Chandler C, Ansah E, Leslie T, Staedke SG. Deployment of ACT antimalarials for treatment of malaria: challenges and opportunities. Malar J. 2008;7(Suppl 1):S7.

16. Mccord GC, Liu A, Singh P. Deployment of community health workers across rural sub-Saharan Africa: financial considerations and operational assumptions. Bull World Health Organ. 2012;91(4):244-253B.

17. Mpembeni RNM, Bhatnagar A, Lefevre A, et al. Motivation and satisfaction among community health workers in Morogoro Region, Tanzania: nuanced needs and varied ambitions. Hum Resour Health. 2015;13:44.

18. Rutta E, Liana J, Embrey M, et al. Accrediting retail drug shops to strengthen Tanzania's public health system: an ADDO case study. J Pharm Policy Pract. 2015;8:23.

19. Goodman CA, Kachur SP, Abdulla S, Bloland P, Mills A. Regulating Tanzania's drug shops - why do they break the rules, and does it matter? Health Policy Plan. 2009;22:393-403.

20. Simba D, Schuemer C, Forrester K, Hiza M. Reaching the poor through community-based distributors of contraceptives: experiences from Muheza district, Tanzania. Tanzan J Health Res. 2011;13(1):1-7.

21. Masanja H, Schellenberg JA, de Savigny D, Mshinda H, Victora CG. Impact of Integrated Management of Childhood Illness on inequalities in child health in rural Tanzania. Health Policy Plan. 2005;20 Suppl 1:i77-84.
22. National Bureau of_Statistics. Tanzania - Population and Housing Census 2012. 2012. Available from: http://www.tzdpg.or.tz/fileadmin/ documents/dpg_internal/dpg_working_groups_clusters/cluster_2/ water/WSDP/Background_information/2012_Census_General_Report. pdf. Accessed October 3, 2018.

23. National Bureau of Statistics. Tanzania HIV/AIDS and Malaria Indicator Survey 2011-12. Dar es Salaam, Tanzania. Available from: https:// dhsprogram.com/pubs/pdf/AIS11/AIS11.pdf. Accessed October 03, 2018.

24. Ministry-of-Health-and-Social-Welfare. Midterm Analytical Review of Performance of the Health Sector Strategic Plan III 2009-2015. 2015. Available from: http://www.who.int/healthinfo/country_monitoring_evaluation/TZ_AnalyticalReport_2013.pdf. Accessed October 3, 2018.

25. Measure-evaluation. Household Survey Indicators for Malaria Control. 2013. Available from: http://www.malariasurveys.org/documents/ Household\%20Survey\%20Indicators\%20for\%20Malaria\%20Control. pdf. Accessed October 3, 2018.

26. Mubiru D, Byabasheija R, Bwanika JB, et al. Evaluation of Integrated Community Case Management in Eight Districts of Central Uganda. PLoS One. 2015;10(8):e0134767.

27. Mubi M, Janson A, Warsame M, et al. Malaria rapid testing by community health workers is effective and safe for targeting malaria treatment: randomised cross-over trial in Tanzania. PLoS One. 2011;6(7):e19753.

28. Marsh VM, Mutemi WM, Muturi J, et al. Changing home treatment of childhood fevers by training shop keepers in rural Kenya. Trop Med Int Health. 1999;4(5):383-389.

29. Gomes MF, Faiz MA, Gyapong JO, et al. Pre-referral rectal artesunate to prevent death and disability in severe malaria: a placebo-controlled trial. Lancet. 2009;373(9663):557-566.

30. Idro R, Marsh K, John CC, Newton CRJ. Cerebral Malaria: Mechanisms of Brain Injury and Strategies for Improved Neurocognitive Outcome. Pediatr Res. 2010;68(4):267-274.

31. Lysack C, Krefting L. Community-based rehabilitation cadres: their motivation for volunteerism. Int J Rehabil Res. 1993;16(2): 133-141.

32. Simba DO, Kakoko DC. Volunteerism among out-of-school adolescent reproductive health peer educators: is it a sustainable strategy in resource constrained countries? Afr J Reprod Health. 2009;13(3):99-110.

33. Kidane G, Morrow RH. Teaching mothers to provide home treatment of malaria in Tigray, Ethiopia: a randomised trial. Lancet. 2000;356(9229):550-555.

34. Kakoko DC, Simba DO, Semali IA, Ezekiel MJ. Strategies and challenges in the provision of health services in context of health service providers' shortage in Tanzania: a qualitative study. Int J Innov Healthc Res. 2013;1:6-14.

35. Ministry-of-Health-and-Social-WelfareWelfare, S. National Guidelines for Diagnosis and Treatment of Malaria. 2006. Available from: http://apps.who.int/medicinedocs/documents/s19271en/s19271 en.pdf. Accessed October 3, 2018.

36. Ministry-of-Health-and-Social-Welfare. Human Resource for Health Country Profile 2012/2013. 2013. Available from: http://www.tzdpg. or.tz/fileadmin/documents/dpg_internal/dpg_working_groups_clusters/cluster_2/health/Key_Sector_Documents/HRH_Documents/ Final_Country_Profile_2013.pdf. Accessed October 3, 2018.

37. WHO Study Group on Community Health Workers \& World Health Organization. Strengthening the performance of community health workers in primary health care : report of a WHO Study Group [meeting held in Geneva from 2 to 9 December 1987]. Geneva: World Health Organization. 1989. Available from: http://www.who.int/iris/ handle/10665/39568. Accessed October 3, 2018. 
Research and Reports in Tropical Medicine is an international, peerreviewed, open access journal publishing original research, case reports, editorials, reviews and commentaries on all areas of tropical medicine, including: Diseases and medicine in tropical regions; Entomology; Epidemiology; Health economics issues; Infectious disease; Laboratory science and new technology in tropical medicine; Parasitology; Public health medicine/health care policy in tropical regions; and Microbiology. The manuscript management system is completely online and includes a very quick and fair peer-review system. Visit http://www.dovepress. com/testimonials.php to read real quotes from published authors.

Submit your manuscript here: https://www.dovepress.com/research-and-reports-in-tropical-medicine-journal 